\title{
An Enhanced Safety System for Auto Mode E-Vehicles through Mind Wave Feedback
}

\author{
Dr. Bindhu V, \\ Professor, Head, \\ Department of Electronics and Communication Engineering, \\ PPG Institute of Technology, \\ Coimbatore, \\ Tamil Nadu, India. \\ Email Id: vbindhuppg@igmail.com
}

\begin{abstract}
Auto mode vehicles are also called as auto pilot vehicles which are running with the help of programmed artificial intelligence. As of now the auto pilot vehicles are designed to operate for certain distance by analyzing the alertness of driver through his physical presence. The physical presence is verified by reading the driver's touch signal to the steering connected in a vehicle. The proposed system is designed to enhance the safety feature of a vehicle by analyzing the driver's alertness through his mind wave. The mind wave signal variations are continuously monitored through a microcontroller and it will enables the auto pilot mode when there is an emergency. The auto pilot mode enabled at such situations are designed to avoid accidents by stopping the vehicle in a safety manner. The prototype of the design is verified in the paper by conducing several experiments.
\end{abstract}

Keywords: E-vehicle safety, auto pilot mode safety, mind wave analyzer, Neurosky mindwave, auto parking.

\section{Introduction}

Cruise control was the first auto option enabled in vehicles to control and maintain the accelerator throttle to certain extent. This improved the convenience of a driver who is running a vehicle for very long distance. But auto pilot mode is a total control operation of a vehicle on its operation. To make this process, several numbers of cameras and sensors are employed in a vehicle. The images and videos captured through such cameras are processed through a microcontroller in a real-time operation for operating a vehicle. Through the cameras, the roadway and opposite vehicle locations are predicted by the computer vision analyzer. From the analyzer the control operations are enabled through a microcontroller. In order to improve the safety features of auto pilot mode, the sensors fixed on a steering wheel are designed to monitor the driver's presence. The signals which are transmitted from the steering wheels at regular intervals enables the driver's presence. Figure 1 indicates the operational block diagram of an auto pilot mode.

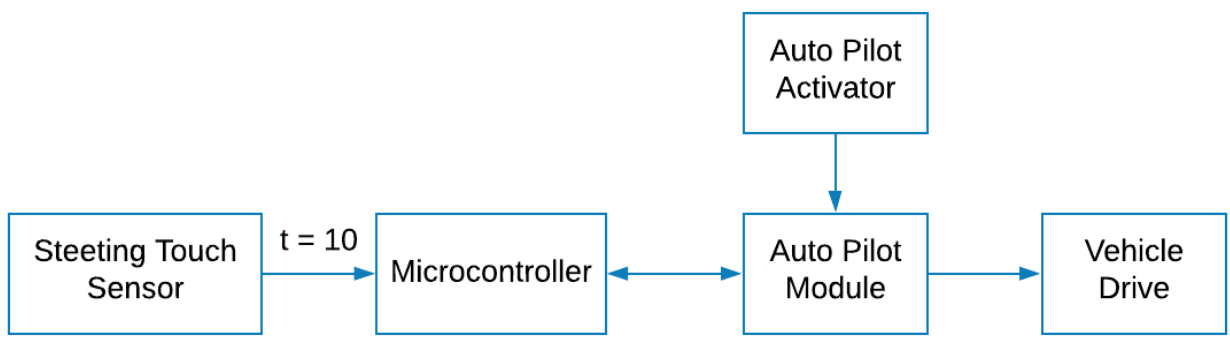

Figure 1. Operational block diagram of general auto pilot mode.

The auto pilot activator is a one touch switch, which enables the microcontroller module designed to drive the vehicle. The switch can be made activated by the driver on steady driving condition. With certain time interval the auto pilot mode will be enabled by the in-built program and a confirmation message will be delivered to the driver 
Journal of Information Technology and Digital World (2020)

Vol.02/ No. 03

Pages: 144-150

https://www.irojournals.com/itdw/

DOI: https://doi.org/10.36548/jitdw.2020.3.002

through the lights available in the instrument cluster. From that time onwards the auxiliary microcontrollers connected with the main controller are enabled to receive signals of driver's presence at regular interval time $(\mathrm{t}=10$ seconds $)$. Figure 2 indicates the blocks available inside the auto pilot module for an overview.

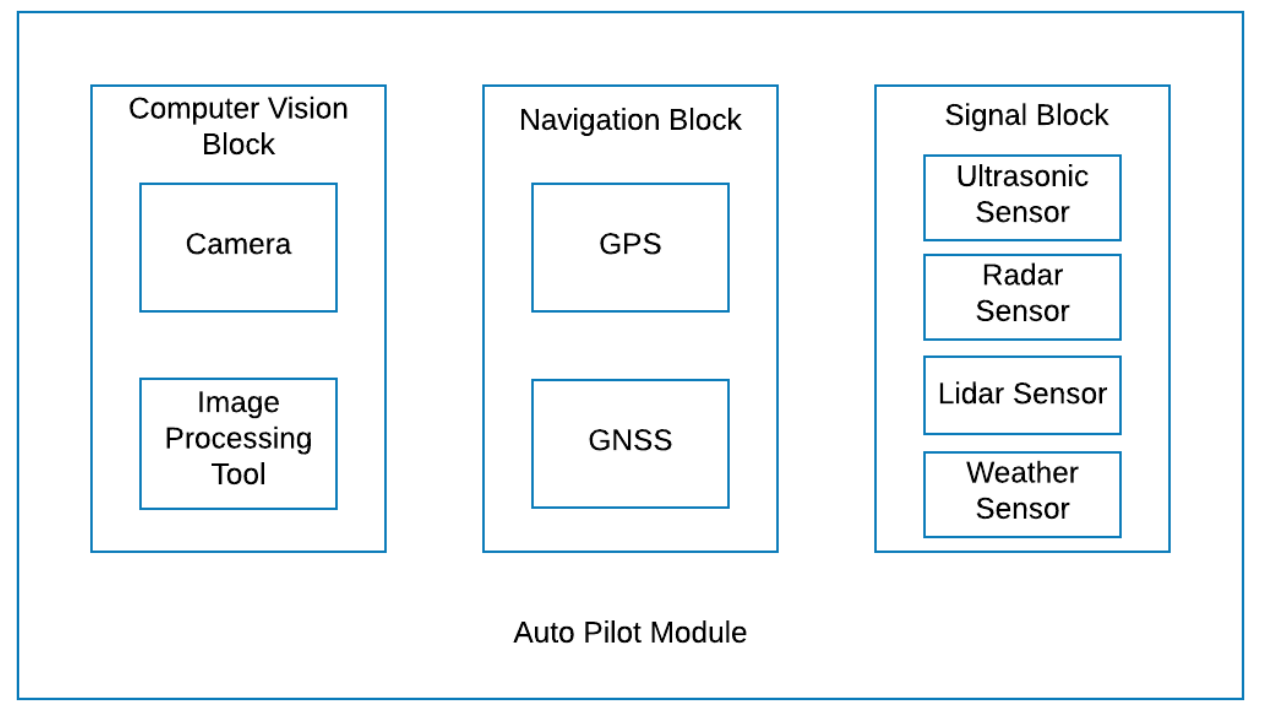

Figure 2. Overview of an Auto Pilot Module.

The auto pilot module can be broadly classified into three blocks as vision block, navigation block and signal block. The vision block consists of several numbers of cameras to capture the 360 degree view from a vehicle. All the image frames captured by the cameras are transferred to an image processing tool to preprocess the images for better computer vision. The deep learning algorithms which are trained to classify several objects and vehicles are kept inside this block to validate the obstacle and moving parts around the vehicle. This make the auto pilot module to get a clear vision about the vehicle surroundings.

Same to as the vision block, the sensor block also enables the auto pilot module to sense the surroundings of a vehicle through the signals received from different kind of sensors. The ultrasonic sensor is a most know sensor used to measure the distance between the obstacles from the sensor through ultrasonic waves. The radar is also a widely used sensor in certain vehicles to identify the obstacles presence in blind spots. The radio waves are acting as a measuring medium in radar devices. The Lidar is also a similar device to radar, which measures the obstacle presence through a light wave transmission. A weather sensor consists of so many sensors are employed in the auto pilot module to measure the climate changes around a vehicle. It helps the auto pilot mode to operate on some specific driving styles. Several other sensors to monitor the road conditions and quality were also used in auto drive cars to ensure the smooth operation of driving.

Global Positioning System (GPS) and Global Navigation Satellite System (GNSS) are helpful in navigation process of an autonomous vehicle. The GPS has the ability to read and transmit the location of the vehicle and similarly the GNSS helps to predict the routes of a destination entered by the driver of a vehicle. The GNSS is more powerful and reliable than the GPS system in terms of accuracy and speed. These three blocks are the peripheral input devices to the auto pilot module. There are several other blocks are there to activate and run the auto pilot modules. All such modules are connected to the motor or engine of the vehicle through a relay circuit or motor driver unit. It varies depends upon the vehicle type and driving conditions. 
Journal of Information Technology and Digital World (2020)

Vol.02/ No. 03

Pages: 144-150

https://www.irojournals.com/itdw/

DOI: https://doi.org/10.36548/jitdw.2020.3.002

\section{Related Work}

A brain machine interfacing system [1] was introduced to control a system by reading the mind wave of a human brain. The electric discharge produced from a human brain on doing some tasks may vary with respect to the number of neurons working on it. The task of the work is to read such electrical signal variations for controlling an interfacing system. The work analyses several applications working towards brain interfacing model. An algorithm for enhancing the safety parameters in a self-driving cars was developed [2] by verifying the existing safety features in real time operations. The proposed algorithm improves the performance of the existing algorithms built inside the microcontroller. A color study experiment was made using mind wave sensor [3] to predict the neurology changes when a human sees some color. The experiment was conducted by showing different shapes in different colors to some number of peoples to say their shape along with its color. An EEG mind wave sensor was also their on such humans head to read the neurological changes from their mind. The result shows that there are some similarity among the neurological signals produced among the people when they sees a same color of shape objects. The study experiment was conducted to find out the concentration level of a person.

A virtual reality simulator [4] was designed to treat patients with post-traumatic stress disorder. Here the people affected with minor stress disorder due to several life incidents are treated through a virtual simulator. The virtual simulator creates the scene intelligently for the people to visualize the incident. At that time hear beat and several neurological changes are observed from the patient for understanding their mind disorders in a precise manner. This improves the treatment to move forward with a healthy practice. An evaluation on bio potential signal acquisition was performed [5] to compare the accessing speed of two different microcontroller architectures. The signals are gathered from a readers mind through Neurosky mind wave device. The device is connected with a Bluetooth transmitter for sending the signal to the destination ports connected with arduino as well as Raspberry pi to evaluate its speed in processing the information. An augmented virtual reality method [6] was developed to predict the archeological content damaged due to several climate changes. The visualization was predicted by creating a three dimensional model for comparing it to the existing damaged structures.

An experiment on brain attention detection [7] was conducted to measure the attention level of humans at various age. The brain attention level was analyzed for men as well as women of different ages to predict their brain attention level. For this an arduino uno microcontroller was employed with a serial monitor to graph the predicted values from the mind wave sensor. The mind wave senor acts as an EEG device to read the mental strength of human by their mind changes. A series of three LEDs was placed in this experiment to visualize the attention level of a human connected with mind wave device. The human attention level can be changed by modulating the written program on the microcontroller. A virtual reality gaming system was designed to predict the user's mental strength [8] through EMG signal measurements. The signals are measured when the user does some specific movements like pedal exercises and so on. The motions are captured through Yei-3 space sensor for transmitting the signals to the processor. A robotic hand control system based on mind wave reading was proposed [9] to control the fingers attached in the robotic hand. The signals captured from Neurosky mind wave generator and transmitted to a LabVIEW application for controlling the peripherals connected with it. The major portion of control is handled by observing the eye blink status of the user connected with the mind wave reader.

A six axis mechanical arm was designed using computer vision technology [10] to recognize the objects for pick and place operation. The operation was handled by training the algorithm through giving samples of different objects to understand the reality. Along with recognition of shapes, a color learning feature was added to the algorithm for predicting the color changes to the shape objects. The accuracy of the proposed technology was ranged between 75 to 89 percentages. A mobile robot was controlled using mind wave generated from a faraway user [11]. The communication between the mind wave sensor and the robot kit was made through Bluetooth communication. There are two microcontrollers were utilized in the project for transmitting the mind wave signal and another one to read and implement the mind wave signal to the robot. The direction of the motors are controlled by the mind waves and a set of LED lights were utilized in this work predict the direction of movement of the connected robot. A Bluetooth

ISSN: 2582-418X

Submitted: 28.07.2020

Accepted: 20.08.2020

Published: 28.08.2020

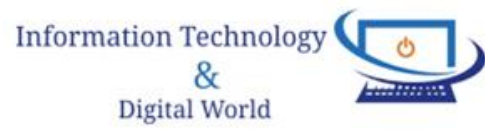


Journal of Information Technology and Digital World (2020)

Vol.02/ No. 03

Pages: 144-150

https://www.irojournals.com/itdw/

DOI: https://doi.org/10.36548/jitdw.2020.3.002

based control system was structured to control the remote placed motor unit [12]. The experiment was conducted with Bluetooth technology along with WiFi unit to calculate the reliability between the two protocols. The experiment result show that the Bluetooth and WiFi are reliable with its specification when there is no interruption. The Bluetooth technology suffers a lot than the WiFi during interruption blockage.

A smart home automation system was proposed to control several home appliances through brain computer interface technology [13]. The brain and computer interface was established by sending the brain signals to the computer through a wireless medium. The signals from the brain are calculated through EEG sensor and the signals are forwarded to the control unit to read and respond to the received signal. This helps several severe injured patients as well as the disabled peoples. The overall circuit and connections are monitored through a Raspberry pi microcontroller kit. A computer vision based human-machine in interaction system was analyzed [14] to understand the capability of the present network structures to replicate the human brain process. The paper undergoes the work carried out by several techniques of deep learning and neural network to remark its achievements and limitations. A neurofeedback system was framed to analyze the concentration level of a reader while reading some article [15]. The system was equipped with a mind wave reader called Neurosky EEG transmitter to measure the changes on readers mind. The signals are transmitted to a microcontroller to display the mental stability on three different LEDs connected with the device. The intensity of the LED light varied when there is a change in mind wave sense.

\section{Proposed Work}

The proposed work is designed to give feedback to the safety system connected in auto mode vehicle from human mind wave signals. The human mind wave is measured here with the help of Neurosky mind wave EEG headset with a Bluetooth transceiver. Figure 3 indicates the connection prototype of the proposed safety system. A DC motor is employed in the prototype to act as an electric drive motor. The manual control module is connected with a computer keyboard to operate as an accelerator of a vehicle. A specific button of the keyboard is assumed as an auto pilot mode selector switch. It enables the microcontroller operation to choose manual drive mode and auto drive mode. In auto drive mode the motor is programmed to run on some specific rpm but in case of manual drive mode the motor is programmed to operate with the switch commands received from the keyboard.

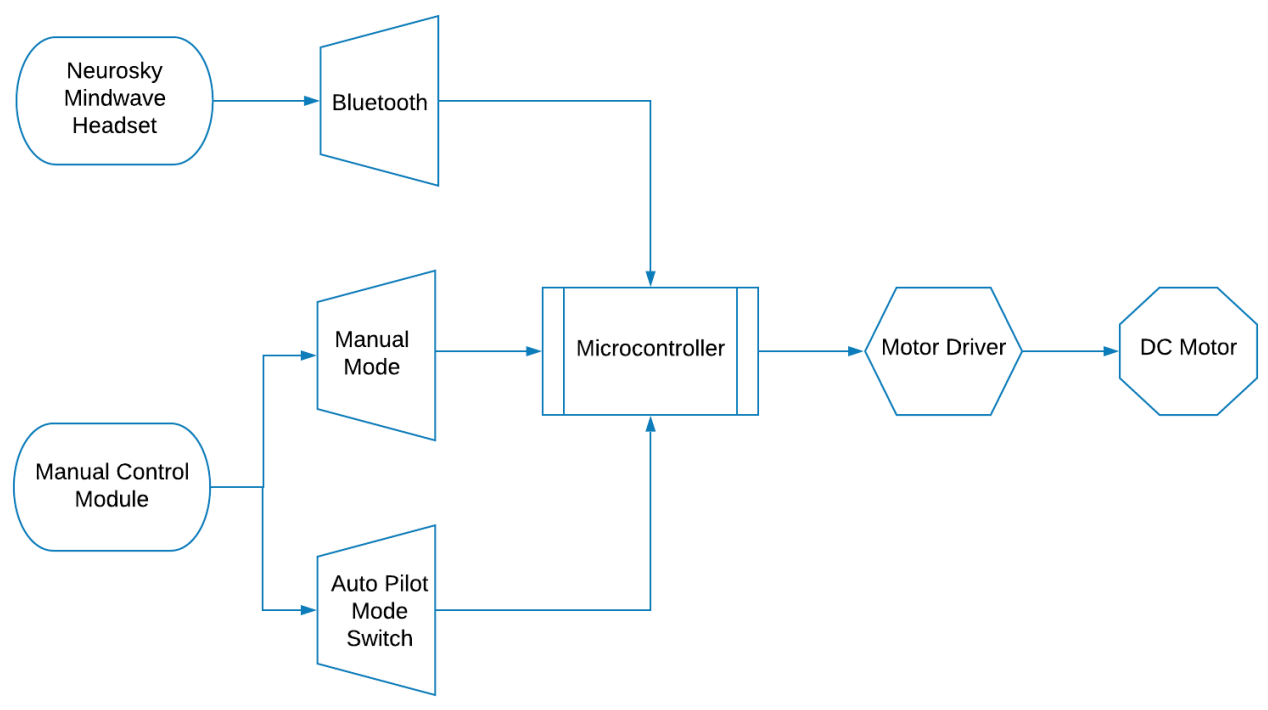

Figure 3. Prototype of the proposed mind wave feedback safety system.

ISSN: 2582-418X

147

Submitted: 28.07 .2020

Accepted: 20.08 .2020

Published: 28.08.2020

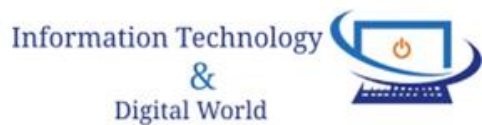


Journal of Information Technology and Digital World (2020)

Vol.02/ No. 03

Pages: 144-150

https://www.irojournals.com/itdw/

DOI: https://doi.org/10.36548/jitdw.2020.3.002

A Neurosky headset is placed over the forehead of the motor operator to read his mind stability. The mind wave reader is attached to a Bluetooth transceiver for transmitting the measured mind wave signal from the Neurosky headset to the microcontroller. An arduino uno microcontroller is utilized in this work for connecting all the four modules. The operations of the proposed model can be modified by changing the eye blink ratio of the operator.

The operation process of the proposed model is shown in figure 4 (b). All the peripherals connected in the proposed system gets activated once the vehicle comes to operation position. Figure 4 (a) represents the operations of the existing auto mode system. The existing model works by getting the feedback signal from the steering wheel sensor. The sensor reads the signal of steering sensor for every 10 seconds in general. With respect to that signal the auto mode action of the vehicle will be continued for next 10 seconds. Similarly the auto mode read switch is continuously monitored by the microcontroller for any change in operation. The limitations and drawbacks of the existing model auto pilot is listed below for better understanding.

- The existing auto pilot mode never stops or parks the vehicle on emergency situation.

- Chances of accident is high due to change of driving mode without driver's attention.

- It reads only physical verification of driver presence.
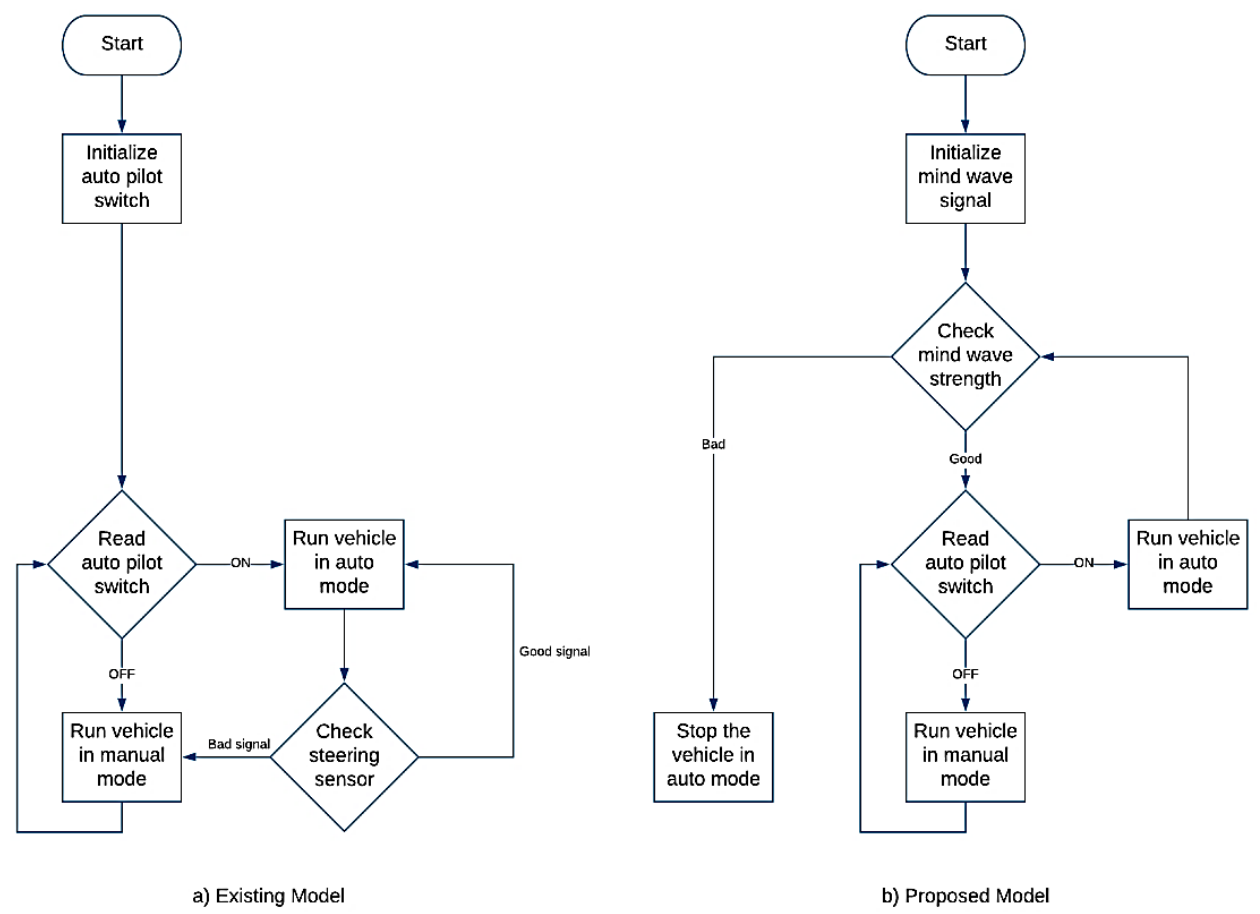

Figure 4. a) Existing model operation, b) proposed model operation.

In the proposed mind wave feedback system the control of the whole vehicle is depends upon the attention level of the driver. The driver attention level is measured continuously with his eye blinking count and interval. The Neurosky mind wave reader has the ability to read the electrical signals produced by the human neurons when they are lazy and in active. These signals are transmitted to the microcontroller to understand that the driver is not in attention mode to continue the journey. So that the microcontroller activates the driving module into automatic mode to park or stop the vehicle from the road in a safe manner. This improved feature enables the vehicle to stop by its own during emergency. The change in mind wave can also be understandable by the proposed method when the driver 
Journal of Information Technology and Digital World (2020)

Vol.02/ No. 03

Pages: 144-150

https://www.irojournals.com/itdw/

DOI: https://doi.org/10.36548/jitdw.2020.3.002

is having a liquor. The only problem in the proposed system is the wireless communication. An improved wireless communication of mind wave signal from the transmitter to the receiver improves the reliability of the work. The proposed model can also modified to visualize the drivers attention to the co-passengers. The attention level of the driver can also be transferred to any other far away destination through adapting an IoT modules. The auto run mode of the vehicle can be extended to continue the journey when the vehicle is having a proper navigation set-up. As of now the commercial available vehicle with auto pilot modes are not having such option. Hence in the proposed mind wave safety system the signals are not considered for navigation process.

\section{Results and Discussion}

The proposed work is verified by connecting the microcontroller to a DC motor for visualizing the working process. The operation is verified with three different peoples with seven different mind state. The measured mind wave frequency is connected to the serial monitor of the arduino microcontroller visualize the output. The following table 1 shows the measured mind wave output in $\mathrm{Hz}$ with motor operation. Similarly, table 2 indicates the general frequency range of various physical condition.

Table 1. Results of the proposed mind wave safety system.

\begin{tabular}{|c|c|c|c|}
\hline Sl. No & $\begin{array}{c}\text { Physical } \\
\text { State }\end{array}$ & $\begin{array}{c}\text { Measured mind wave } \\
\text { frequency in Hz }\end{array}$ & Motor working condition \\
\hline Person 1 & Active & 18 & Manual \& Auto mode \\
\hline Person 2 & Relaxed & 12 & Manual mode only \\
\hline Person 3 & Active & 27 & Manual \& Auto mode \\
\hline Person 4 & Drowsiness & 4 & Stopped in Auto mode \\
\hline Person 5 & Drowsiness & 6 & Stopped in Auto mode \\
\hline Person 6 & Active & 33 & Manual \& Auto mode \\
\hline Person 7 & Relaxed & 7 & Manual mode only \\
\hline
\end{tabular}

Table 2. General frequency emission of mind changes.

\begin{tabular}{|c|c|}
\hline Mind condition & Mind wave frequency range \\
\hline Deep sleep & $0.4-4 \mathrm{~Hz}$ \\
\hline Drowsiness & $4-8 \mathrm{~Hz}$ \\
\hline Relax & $8-14 \mathrm{~Hz}$ \\
\hline Active & $14-35 \mathrm{~Hz}$ \\
\hline
\end{tabular}

The physical state of the persons involved in verifying the work is engaged with different activities to observe the success rate of the proposed algorithm. The active people in table 1 are made to be engaged with simulation driving games, cycling and chess gaming. The relaxed people are encouraged to listen music and watch videos. The drowsiness people are verified immediate after a heavy meal in their nap time. From the results it is clear that the proposed prototype was working fine with the desired output. The change in operations are happened immediately after observing the frequency levels. The experimental tests were also conducted by giving certain delay in changing the mode condition of the vehicle. The only limitation of the proposed work is wireless communication. All the tests were experimented with 2 to 3 feet distance only. The Bluetooth communication and pairing took little bit time to connect the mind wave generator with the connected circuit. For real time operations the wireless communication has to be made connect in automatic mode. Also the pairing signal and condition has to be notified to the driver for ensuring the safety level of the ongoing vehicle. 
Journal of Information Technology and Digital World (2020)

Vol.02/ No. 03

Pages: $144-150$

https://www.irojournals.com/itdw/

DOI: https://doi.org/10.36548/jitdw.2020.3.002

\section{Conclusion}

The physical presence of the driver in the existing model auto cars are observed from the touch sensor connected with steering system. The proposed model suggests to observe the mental presence of the driver while driving a vehicle. A mind wave reader using Bluetooth communication was employed in the prototype to verify the proposed design. The vehicle movement is assumed with a DC motor operation connected to the prototype. The mind wave changes are considered by changing the mindset of the verifiers by giving various tasks. From the connected people mind wave the motor running condition was analyzed in the experimental process of the proposed design. The mind wave feedback can give efficient output to understand the physical and mental presence of a driver while driving for long hours. The proposed design can be implemented in a real time vehicle with very good communication medium.

\section{References}

1. Kounte, Manjunath R., Pratyush Kumar Tripathy, P. Pramod, and Harshit Bajpai. "Implementation of Brain Machine Interface using Mind wave Sensor." Procedia Computer Science 171 (2020): 244-252.

2. Manoharan, Samuel. "An improved safety algorithm for artificial intelligence enabled processors in self driving cars." Journal of Artificial Intelligence 1, no. 02 (2019): 95-104.

3. Teixeira, Ana Rita, and Anabela Gomes. "A Study of Colour Using Mindwave EEG Sensor." In International Conference on Human-Computer Interaction, pp. 176-188. Springer, Cham, 2020.

4. $\quad$ Smys, S., and Jennifer S. Raj. "Virtual Reality Simulation as Therapy for Posttraumatic Stress Disorder (PTSD)." Journal of Electronics 1, no. 01 (2019): 24-34.

5. Galíndez-Floréz, Iván, Andrés Coral-Flores, Edna Moncayo-Torres, Dagoberto Mayorca-Torres, and Herman Guerrero-Chapal. "Biopotential Signals Acquisition from the Brain Through the MindWave Device: Preliminary Results." In International Conference on Applied Technologies, pp. 139-152. Springer, Cham, 2019.

6. Shakya, Subarna. "Virtual Restoration of Damaged Archeological Artifacts Obtained From Expeditions using 3D Visualization." Journal of Innovative Image Processing (JIIP) 1, no. 02 (2019): 102-110.

7. Pari-Larico, Susan, Brandon Llerena-Urday, Álvaro Fernández del Carpio, Karina Rosas-Paredes, and José Esquicha-Tejada. "Evaluation of Brain Attention Levels Using Arduino and Neurosky Mindwave EEG According to Age and Sex." (2019).

8. Smys, S. "Virtual reality gaming technology for mental stimulation and therapy." Journal of Information Technology 1, no. 01 (2019): 19-26.

9. Ruşanu, Oana Andreea, Luciana Cristea, Marius Cristian Luculescu, and Sorin Constantin Zamfira. "Experimental Model of a Robotic Hand Controlled by Using NeuroSky Mindwave Mobile Headset." In 2019 E-Health and Bioengineering Conference (EHB), pp. 1-4. IEEE, 2019.

10. Chen, Joy Iong-Zong, and Jen-Ting Chang. "Applying a 6-axis Mechanical Arm Combine with Computer Vision to the Research of Object Recognition in Plane Inspection." Journal of Artificial Intelligence 2, no. 02 (2020): 77-99.

11. Dragomir, Florin, Mihai Stefan Caramida, Otilia Elena Dragomir, and Eugenia Minca. "Towards neural control of the mobile robots." Journal of Science and Arts 19, no. 2 (2019): 529-540.

12. Vinothkanna, Mr R. "Design and Analysis of Motor Control System for Wireless Automation." Journal of Electronics 2, no. 03 (2020): 162-167.

13. Narudin, Siti Khadijah. "Smart Home Control for Disabled Using Brain Computer Interface." International Journal of Integrated Engineering 12, no. 4 (2020): 74-82.

14. Suma, V. "Computer Vision for Human-Machine Interaction-Review." Journal of trends in Computer Science and Smart technology (TCSST) 1, no. 02 (2019): 131-139.

15. Lochotinunt, Chanin, Nattasasi Suwanpathumlert, Nattachai Masawat, Surapan Airphaiboon, and C. Pintavirooj. "Neurofeedback System." In 2019 12th Biomedical Engineering International Conference (BMEiCON), pp. 16. IEEE, 2019. 